\title{
Surgical treatment of primary tracheal tumors in children and adolescents
}

\author{
Lorenzo Giannini ${ }^{1,2}$, Fabiola Incandela ${ }^{1}$, Lorenzo Bresciani ${ }^{1}$, Cesare Piazza ${ }^{1}$ \\ ${ }^{1}$ Department of Otorhinolaryngology, Maxillofacial, and Thyroid Surgery, Fondazione IRCCS, National Cancer Institute of Milan, University of \\ Milan, Milan, Italy; ${ }^{2}$ Departmemnt of Otorhinolaryngology-Head and Neck Surgery, ASST Santi Paolo e Carlo, University of Milan, Milan, Italy \\ Contributions: (I) Conception and design: All authors; (II) Administrative support: C Piazza; (III) Provision of study materials or patients: None; (IV) \\ Collection and assembly of data: L Giannini, F Incandela, L Bresciani; (V) Data analysis and interpretation: All authors; (VI) Manuscript writing: All \\ authors; (VII) Final approval of manuscript: All authors. \\ Correspondence to: Cesare Piazza, MD. Chief of the Department of Otorhinolaryngology, Maxillofacial, and Thyroid Surgery, Fondazione IRCCS, \\ National Cancer Institute of Milan, University of Milan, 20133 Milan, Italy. Email: cesare.piazza@istitutotumori.mi.it; ceceplaza@libero.it.
}

\begin{abstract}
Pediatric primary tracheal tumors (PTTs) are rare entities, with an estimated incidence of $0.2 \%$ among all neoplasms in children and adolescents. Benign lesions and malignancies are rather equally represented, with subglottic hemangioma, granular cell tumor, carcinoid tumor, and low-grade mucoepidermoid carcinoma being the most common histotypes. Guidelines on management and treatment of pediatric PTTs are not available, and only a few reviews, case reports, and single surgeon/institute experiences have been published. A literature search was carried out focusing on the surgical approaches utilized in the most recent publications to manage pediatric PTTs, thus including case reports, single institute experiences, and reviews on surgical techniques. Moreover, we divided the available intervention modalities based on an anatomical classification, distinguishing between laryngotracheal, cervical tracheal, lower tracheal, and carinal scenarios. Endoscopic treatment is still underused, even for benign lesions: this is mainly due to the difficulties that surgeons may experience during airway management, appropriate tumor visualization, and its safe removal with adequate prevention/management of intraoperative complications. Considering open surgery, simple tumorectomy via median thyrotomy is the treatment of choice whenever possible (mainly for benign tumors), whereas in case of wider excisions, laryngotracheal resection and reconstruction with cartilage graft, end-to-end anastomosis, or neo-carinal reconstruction represent the most widely used procedures. All these techniques are similar to what described in adults. Strict cooperation with an experienced team of pediatric anesthesiologists and intensivists is of paramount importance for the adequate management of PTTs in young children. We are far from establishing a common guideline for treatment of PTTs. Mini-invasive procedures should be encouraged and developed since the majority of pediatric histotypes are benign or low-grade cancers. Moreover, patients should be addressed to specialized pediatric centers in order to improve and refine the existing techniques, laying the groundwork for the proposal of new procedures.
\end{abstract}

Keywords: Adolescents; children; primary tumors; surgical management; tracheal tumors

Received: 18 February 2019; Accepted: 25 February 2019; Published: 06 March 2019.

doi: $10.21037 / \mathrm{pm} .2019 .03 .01$

View this article at: http://dx.doi.org/10.21037/pm.2019.03.01

\section{Introduction}

Pediatric primary tracheal tumors (PTTs) are extremely uncommon entities, even when extending the literature review to also include tumors affecting adolescents up to the 18th year of age. The effective prevalence of these lesions remains unclear, since most reviews tend to collect laryngotracheal, tracheobronchial, and endobronchial neoplasms together, making it difficult to extrapolate the 


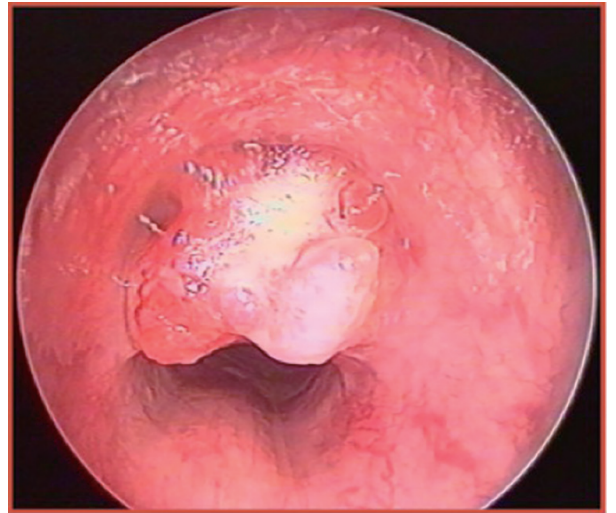

Figure 1 An asymptomatic 14-year-old girl had been intubated to be submitted to tonsillectomy under general anaesthesia in another hospital. After extubation, she presented mild dyspnea and hemoptysis. At fibreoptic examination of the upper aerodigestive tract, bleeding was seen arising from the trachea. Subsequent bronchoscopy confirmed the presence of a tumor involving less than $50 \%$ of the airway lumen, originating from the anterior wall of the trachea, and apparently extending from the second to the third tracheal ring. CT scan confirmed the cranio-caudal extent of the lesion and ruled out any extra-mural involvement of adjacent cervical organs. Neodymium yttrium aluminum garnet laserassisted endoscopic debulking of the tumor was performed and a diagnosis of low-grade mucoepidermoid carcinoma obtained. Tracheal resection of the first 4 tracheal rings with subsequent crico-tracheal anastomosis within free surgical margins was subsequently performed through a standard cervicotomic approach. The patient was extubated immediately after surgery and no postoperative complications occurred. She was discharged at 14 days after surgery with normal airway and diet. She is endoscopically and radiologically without evidence of disease at 18 years after surgery.

component purely related to PTTs. With such a caveat in mind, it seems reasonable to estimate that only $8 \%$ of PTTs occur in this age group, with an incidence possibly around $0.2 \%$ of all pediatric neoplasms (1-3). Moreover, due to the rarity of the disease, there is still no consensus on the relative prevalence of benign versus malignant forms in the pediatric-adolescent population: in fact, in 1998, Desai et al. conducted a literature review of more than 100 publications, and found that $64 \%$ of tracheal tumors in patients under 18 years of age were benign, while the remaining were cancers (4). By contrast, Roby et al. found the opposite proportion ( 2 benign for every 3 malignant PTTs) in a more recent single-institute case series (5).
For what concerns the different histopathological entities described to date, recurrent respiratory papillomatosis (RRP) is considered the most common benign subglottic and tracheal tumor in the pediatric age. However, due to its human papilloma virus-related etiology and peculiar biologic behavior, tracheal RRP presents specific challenges and clinical issues that make it wise to classify and discuss it separately, mainly as a complication/sequela or late evolution of the much more common laryngeal RRP. For this reason, the present review will not focus on tracheal RRP. In PTTs, however, the histotypes most frequently reported in the literature are hemangioma, granular cell tumor, inflammatory myofibroblastic tumor, mucous gland tumor, juvenile xanthogranuloma, chondroma, neurofibroma, pleomorphic adenoma, and lipoblastoma $(4,6)$.

Regarding the malignant forms, many authors have reported that carcinoid tumor is the most frequent PTT, followed by mucoepidermoid carcinoma (MEC) (Figure 1). Altogether, these two nosologic entities represent more than $80 \%$ of all pediatric endotracheal malignancies $(5,7)$. However, fortunately enough, both tumors present a lower recurrence rate and better prognosis than the corresponding adult forms (3). Fibrosarcoma, rhabdomyosarcoma, adenoid cystic carcinoma, bronchogenic carcinoma, and leiomyosarcoma are less common $(3,4)$, and their site of origin has been frequently shown to be the posterior tracheal wall (4).

Wheezing and stridor constitute the most common presentation in PTTs, followed by cough and pneumonia $(4,7,8)$. For this reason, the most frequent misdiagnosis of PTTs in this age cohort is asthma: the ensuing inappropriate use of bronchodilators may partially mask, at least at beginning, a diagnosis of tumor and delay its correct surgical treatment in a significant percentage of cases (1). By contrast, dyspnea as an initial symptom is rather uncommon, since it develops only when the tumor significantly reduces tracheal lumen patency to less than $50 \%$ (1). On the other hand, hemoptysis appears when the overlying mucosa becomes ulcerated and is rare in benign tumors, being mainly described in MEC $(6,8)$. In case of carcinoid tumors, carcinoid syndrome with hypotension, diarrhea, and flushing has been described in $30 \%$ of cases, in various association with non-specific endoluminal massrelated signs and symptoms (6).

Even though radical surgery of PTTs remains the treatment of choice whenever feasible, there are still no guidelines about the most adequate technique to be adopted, and in the literature only a limited number of reviews and case reports related to a single intervention or 
single institution case series are present. As in most rare and orphan diseases, expert opinion is of paramount importance and can be the only reasonable substitute to the shortage of large retrospective and prospective trials.

The aim of this review is therefore to present the state of the art of surgical approaches to PTTs in children and adolescents, dividing the available techniques according to anatomical classification.

\section{Surgical treatment}

\section{Tracbeotomy}

While at clinical presentation in adults PTTs may reduce the airway up to $75 \%$ of its lumen frequently without major airway problems, in children the asymptomatic average obstruction is slightly inferior, usually no more than $50 \%$ $(1,4)$. Nevertheless, pediatric patients may experience respiratory distress at presentation or during fiberoptic examination due to a bulky lesion with superimposition of a concomitant airway infection or ulceration, bleeding, and ensuing edema. In this case, some type of emergent airway measure must be taken before the appropriate diagnostic work-up has been completed and tumor resection adequately planned (6). Even though endoscopic debulking would be definitely preferable for its reduced morbidity, the luxury of performing simultaneous bioptic sampling of the lesion and, most importantly, no consequent preclusion to further surgical procedures, this requires considerable expertise, adequate facilities, and a pediatric team used to work in emergency situations, something which is unfortunately not available in all settings. Therefore, the most frequently applied strategy to relieve airway distress in a child affected by PTT, even before getting an adequate diagnosis, is to perform a straightforward emergent tracheotomy. In any case, tracheotomy in this situation should be performed by paying attention to not enter the tumor (for the risk of neoplastic seeding) or making the tracheotomic fenestration too far away (caudally) from the lesion itself. In fact, at definitive surgery, the stoma should be always removed together with the tracheal tract infiltrated by the tumor (to possibly enhance oncological radicality), and this may become no more feasible if tracheotomy has been performed not taking into account these considerations $(3,6)$.

\section{Endoscopic treatment}

The literature about endoscopic treatment of pediatric
PTTs is understandably poor. For sure, however, endoscopic approaches may play multiple roles in management of PTTs, from initial diagnostic evaluation of its precise superficial extent, cranio-caudal length, and histopathological nature, to symptom relief by tumor debulking and, when feasible, radical excision of the lesion itself.

Benign tumors can obviously be more safely removed than malignant ones through an endoscopic approach. For example, subglottic hemangioma (SGH), which represents one of the most common laryngotracheal tumors in the pediatric population, may be definitely managed in this way, even due to its well-known tendency towards progressive spontaneous shrinkage when the child grows into adolescence (9). Moreover, the most modern tendency in management of such a tumor consists in the intravenous administration of propranolol, a medication of the beta blocker class. A recent review of the literature by Schwartz et al. in 2017 found a success rate of $88 \%$ among 49 patients treated in this way for SGH. The remaining $12 \%$ were treated with endoscopic debulking followed by open surgery (10). The same report documented a very low grade of rebound growth after drug administration (9\%), and half of children experiencing such a problem recovered after having increased the pharmacological dosage (10). Therefore, endoscopic and/or open-neck surgical treatment of SGH should be nowadays considered as the last option and performed only after failure of appropriate conservative medical treatment.

Even though carbon dioxide $\left(\mathrm{CO}_{2}\right)$ laser is the most common cutting tool in transoral laryngeal surgery, it is actually less suitable for tracheal or carinal lesions. In fact, even though $\mathrm{CO}_{2}$ laser is now deliverable via optical fibers, its coagulation properties and maneuverability within a laser bronchoscope are suboptimal compared with the superior performance of other tools like potassium titanyl phosphate or neodymium yttrium aluminum garnet lasers, whose wavelengths are more indicated for reducing profuse bleeding from highly vascularized lesions such as SGHs $(9,11)$.

Another tumor which seems to have favorable outcomes when removed through a fiberoptic laser-assisted endoscopic route is fibrous histiocytoma that, in spite of its malignant nature, within the trachea usually presents a benign-like behavior, thus justifying a conservative approach instead of an open procedure (12).

Endoscopic laser-assisted procedures may even assist in performing symptomatic debulking of tracheal masses in case of unresectable lesions, but have also been employed for temporary management of benign or low-grade 
tumors in order to delay definitive open surgery, allowing children to grow and therefore possibly reducing intra- and postoperative complication rates $(8,13)$.

Nevertheless, although endoscopic treatment remains a minimally-invasive procedure, many complications may occur during and after it, e.g., transmural injury, inability to stop important hemorrhage, synechia, and tracheal stenosis. For this reason, the surgeon should carefully evaluate the type and modality of laser delivery in order to maximally preserve tracheal structures, simultaneously preventing undesired complications such as excessive bleeding or tracheal thermal damage with subsequent perforation. Moreover, previous surgeries may generate scar tissues and make the endoscopic approach more challenging (14). Finally, when the tumor presents an aggressive nature, with involvement of the underlying cartilage and/or surrounding cervico-mediastinal soft tissues, endoscopy should be limited to diagnostic evaluation and staging of the endoluminal tumor boundaries before planning open surgery.

\section{Open surgery of tumors involving the larynx}

Once absolute contraindications to radical surgical resection (involvement of more than $50 \%$ of the tracheal length, invasion of mediastinal unresectable organs, massive nodal involvement or distant metastasis in squamous cell carcinoma or other aggressive histotypes) have been ruled out, the clinician should plan the intervention according to the optimal approach, depending mainly on the tumor site and its cranio-caudal and transmural extensions (1).

Whenever the lesion is located within the larynx and/ or cervical trachea, a standard "collar" incision with or without manubriotomy is the most frequently preferred approach, whereas in tumors with lower localization median sternotomy or right posterolateral thoracotomy are usually the most indicated (1).

After skin flap elevation in a subplatysmal plane, strap muscle division along the midline and lateral retraction, the cervical trachea and crico-tracheal junction are exposed after thyroid gland isthmus division $(6,15)$. If the tumor is close to the vocal cords in the posterior aspect of the cricoid plate and its nature is benign or malignant lowgrade, then a median vertical thyrotomy can be performed in order to gain access to this anatomical site and obtain optimal exposure of the tumor (14). To achieve this, the endotracheal tumor should be first removed by performing a lower temporary tracheotomy. In this way, it is possible to accurately inspect the state of cartilaginous infiltration: in fact, if the thyroid and/or cricoid framework is not involved by the tumor, the surgeon may perform a radical tumorectomy and then close the median thyrotomy without the need for further reconstruction $(6,14)$.

On the other hand, in case of high-grade lesions or other tumors deeply involving the laryngeal skeleton, then partial laryngotracheal resection (LTR) and subsequent primary reconstruction should be taken into account (16). This technique consists of splitting the cricoid arch and plate after its partial removal with subsequent increase of the subglottic lumen by the interposition of an autologous cartilage graft. Such a graft may be inserted posteriorly, anteriorly, or at both levels in case of larger defects (16). Even though several donor sites have been employed for harvesting the graft in case of LTR (thyroid lamina, auricular or septal cartilages, hyoid bone), costal cartilage is considered the best option, due to its physical properties, quantity, reliability, and relative paucity of side effects at the donor site (16). Even though the aforementioned technique had been proposed mainly for benign laryngotracheal stenoses and/or congenital malformations, Pernas and coworkers described the role of LTR in a 11-year-old boy affected by granular cell tumor of the inter-arytenoid area, after failure of a previous endoscopic approach (14). The cartilage graft employed in that specific case was of costal origin (14).

If the lesion does not allow an oncologically safe LTR, then crico-tracheal resection (CTR) with crico-tracheal or thyro-crico-tracheal anastomosis should be considered (3). This technique allows radical removal of large tumors, potentially affecting around $50 \%$ of the airway length and its full circumference with intact crico-arytenoid joints. After CTR, children should be kept sedated and intubated in the intensive care unit for at least 5-6 days to prevent cough and neck movements with possible anastomotic dehiscence. Older and more cooperative patients may be extubated sooner, possibly in the operatory room, just at the end of surgery, in order to avoid the presence of secretions above the cuffs and decubitus of the endotracheal tube in the subglottic and inter-arytenoid area. As an alternative, in case of quite cranial resection with possible vocal cords edema, a temporary tracheotomy $2-3$ tracheal rings caudally from the anastomotic line should be performed and removed as soon as the laryngeal airway has been restored in its full patency.

However, close cooperation between the airway surgeon and an experienced team of pediatricians, anesthesiologists, and pediatric intensivists cannot be overemphasized during 
all these procedures since around $20 \%$ of perioperative complications are related to management of the endotracheal tube within the operated larynx and trachea (17).

\section{Open surgery of tumors involving the cervical trachea}

When the tumor is located immediately below the cricotracheal junction, surgeons should assess if the resection may be radical by sparing the cricoid cartilage or if a formal CTR may be requested. In both cases, attention must be paid during the isolation of the trachea from the esophagus, in order to preserve function of both recurrent laryngeal nerves (RLNs) (18).

Whether the planned resection requires cricoid cartilage removal, then proper dissection of the cricothyroid muscles is needed, starting from the midline and then reflecting them laterally over the cricothyroid joints (16). The access to the airway should start superiorly at the level of the inferior margin of the thyroid cartilage, going laterally and turning downward just anteriorly to the cricothyroid joints (16). Once tumor resection has been performed, frozen sections should be sent for surgical margin assessment before accomplishing the anastomotic suture. Anastomosis should be tensionless and, therefore, every possible release maneuver (at the laryngeal as well as at the mediastinal extremes) should be accomplished to reach such a target. Once the approximation of the two tracheal or laryngotracheal stumps is tested, the anastomosis should be performed by radially disposed, interrupted, extramucosal, absorbable stitches, as proposed by Grillo, making sure to obtain a full mucosal approximation, in order to avoid intervening granulations and/or stenosis (19). In the last decades, several materials have been proposed to guarantee a lower rate of anastomotic dehiscence (usually around 5\% in the most representative and modern series), but a recent study did not find significant differences among them, with polyglactin (commercialized as "Vycril") the most widely used (20).

\section{Open surgery of tumors involving the thoracic trachea and carina}

Management of tumors arising from the lower half of the trachea up to the carina may be much more demanding for both the patient and surgical team. In fact, the literature demonstrates a higher postoperative mortality rate $(6-12 \%)$, as reported by Lanuti et al:: this is due to the more invasive approaches used, the frequent need to ventilate only one pulmonary hemisystem, or to use a cardiac bypass $(21,22)$. When the lesion is located behind the manubrium, a transverse cervical incision with a manubriotomy may be necessary, whereas for lower tumors there is no unanimous agreement about which surgical access should be preferred, considering sternotomy or right posterolateral thoracotomy $(6,22)$.

In terms of surgical techniques, those usually employed in children and adolescents do not differ from those described for adult patients. Some authors are more prone to carinal reconstruction in children, due to the flexibility and elasticity of younger cartilaginous structures $(23,24)$. Similar to what already seen in the CTR techniques described above, in carinal resection and tracheobronchial reconstruction excessive anastomotic tension must also be avoided: in order to reach adequate release, the surgeon should create a pretracheal plane of dissection from below and perform the mobilization of the hilum with an inferior hilar release from either side (25). Once adequate mobility of the tracheobronchial structures has been obtained, reconstruction is tailored depending on the width of the surgical defect. For small tumors involving the carina, the left and right mainstem bronchi can be sutured to the distal carina to create a "neo-carina" (25). Stieglitz and coworkers described this type of reconstruction in a case of carinal granular cell tumor in a 14-year-old girl (26).

In case of longer tracheal resection, the left bronchus should be attached to the trachea with an end-to-end anastomosis, while the right one is sutured to the trachea by an end-to-side anastomosis. Moreover, in case of massive tracheal involvement, carinal reconstruction may consist in an end-to-end anastomosis between the trachea and right main bronchus, with the left one sutured with an end-to-side anastomosis to the intermedius bronchus (25). The latter technique was reported by Hoseok and coworkers in 2005 after resection of a carinal inflammatory myofibroblastic tumor through right posterolateral thoracotomy in a 4-year-old child, obtaining a satisfying oncologic and functional outcome (24). Finally, in order to ensure a lower rate of anastomotic dehiscence, autologous tissue such as pleura or pedicled pericardial fat flap should be used to wrap the suture line (25). Pericardial fascia can be used as well, both as external reinforcement of the anastomosis and as a reconstructive patch for minor tracheal defects (15).

\section{Conclusions}

Pediatric PTTs are rather uncommon pathologic entities, 
rarely encountered also by airway surgeons performing these kinds of procedures in the general population with a high frequency. Because of their rarity, it is difficult to establish unanimously approved treatment guidelines or speculate on oncological outcomes apart from what deducible by anecdotal experiences and limited case series. From a technical point of view, the surgical procedures available today mainly resemble those proposed in the adults. Due to the high rate of benign lesions and low-grade malignancies, mini-invasive procedures such as endoscopic approaches should be preferred, whenever feasible. Nonetheless, due to the challenging nature of this clinical scenario, centralization of pediatric PTTs is mandatory, in order to allow specialized and designated medical teams to gain experience, improve their skills, optimize treatment outcomes, and refine new techniques to propose to the medical community.

\section{Acknowledgements}

None.

\section{Footnote}

Conflicts of Interest: The authors have no conflicts of interest to declare.

\section{References}

1. Macchiarini P. Primary tracheal tumours. Lancet Oncol 2006;7:83-91.

2. Eyssartier E, Ang P, Bonnemaison E, et al. Characteristics of endobronchial primitive tumors in children. Pediatr Pulmonol 2014;49:E121-5.

3. Varela P, Pio L, Brandigi E, et al. Tracheal and bronchial tumors. J Thorac Dis 2016;8:3781-6.

4. Desai DP, Holinger LD, Gonzalez-Crussi F. Tracheal neoplasms in children. Ann Otol Rhinol Laryngol 1998;107:790-6.

5. Roby BB, Drehner D, Sidman JD. Pediatric tracheal and endobronchial tumors: an institutional experience. Arch Otolaryngol Head Neck Surg 2011;137:925-9.

6. Varela P, Pio L, Torre M. Primary tracheobronchial tumors in children. Semin Pediatr Surg 2016;25:150-5.

7. Jaramillo S, Rojas Y, Slater BJ, et al. Childhood and adolescent tracheobronchial mucoepidermoid carcinoma (MEC): a case-series and review of the literature. Pediatr Surg Int 2016;32:417-24.
8. Lin $\mathrm{CH}$, Chao $\mathrm{YH}, \mathrm{Wu} \mathrm{KH}$, et al. Primary mucoepidermoid carcinoma at the carina of trachea presenting with wheezing in an asthmatic child mimicking an attack of asthma: A case report. Medicine (Baltimore) 2016;95:e5292.

9. Rameau A, Zur KB. KTP laser ablation of extensive tracheal hemangiomas. Int J Pediatr Otorhinolaryngol 2011;75:1200-3.

10. Schwartz T, Faria J, Pawar S, et al. Efficacy and rebound rates in propranolol-treated subglottic hemangioma: A literature review. Laryngoscope 2017;127:2665-72.

11. McQueen CT, Cullen RD. Endoscopic ablation of distal tracheal lesions using Nd:YAG contact laser. Int J Pediatr Otorhinolaryngol 2003;67:181-3.

12. Conforti S, Bonacina E, Ravini M, et al. A case of fibrous histiocytoma of the trachea in an infant treated by endobronchial ND:YAG laser. Lung Cancer 2007;57:112-4.

13. Pfeiffer M, Cohn JE, Pascasio JM, et al. Treatment of an obstructive, recurrent, syncytial myoepithelioma of the trachea with tracheal resection and reconstruction. Int $\mathrm{J}$ Pediatr Otorhinolaryngol 2018;109:85-8.

14. Pernas FG, Younis RT, Lehman DA, et al. Management of pediatric airway granular cell tumor: role of laryngotracheal reconstruction. Int J Pediatr Otorhinolaryngol 2006;70:957-63.

15. Backer CL, Mavroudis C, Gerber ME, et al. Tracheal surgery in children: an 18-year review of four techniques. Eur J Cardiothorac Surg 2001;19:777-84.

16. Monnier P. editor. Pediatric Airway Surgery: Management of Laryngotracheal Stenosis in Infants and Children. 1st edition. Springer-Verlag Berlin Heidelberg, 2011.

17. Younis RT, Lazar RH, Astor F. Posterior cartilage graft in single-stage laryngotracheal reconstruction. Otolaryngol Head Neck Surg 2003;129:168-75.

18. Dimachkieh AL, Dobbie A, Olson DR, et al. Tracheal paraganglioma presenting as stridor in a pediatric patient, case report and literature review. Int J Pediatr Otorhinolaryngol 2018;107:145-9.

19. Grillo HC. Tracheal tumors: surgical management. Ann Thorac Surg 1978;26:112-25.

20. Schilt PN, McRae BR, Akkus O, et al. An experimental model to investigate initial tracheal anastomosis strength. Laryngoscope 2010;120:1125-8.

21. Lanuti M, Mathisen DJ. Carinal resection. Thorac Surg Clin 2004;14:199-209.

22. Romão RL, de Barros F, Maksoud Filho JG, et al. Malignant tumor of the trachea in children: diagnostic 
pitfalls and surgical management. J Pediatr Surg 2009;44:e1-4.

23. Kim J, Park C, Kim K, et al. Surgical resection of mucoepidermoid carcinoma at the carina in a 9-year-old boy. J Pediatr Surg 1998;33:1561-2.

24. Hoseok I, Joungho H, Ahn KM, et al. Complete surgical resection of inflammatory myofibroblastic tumor with

doi: $10.21037 / \mathrm{pm} .2019 .03 .01$

Cite this article as: Giannini L, Incandela F, Bresciani L, Piazza C. Surgical treatment of primary tracheal tumors in children and adolescents. Pediatr Med 2019;2:3. carinal reconstruction in a 4-year-old boy. J Pediatr Surg 2005;40:e23-5.

25. Mitchell JD, Mathisen DJ, Wright CD, et al. Clinical experience with carinal resection. J Thorac Cardiovasc Surg 1999;117:39-52.

26. Stieglitz F, Kitz R, Schäfers HJ, et al. Granular cell tumor of the trachea in a child. Ann Thorac Surg 2005;79:e15-6. 